\title{
Eops: Estenosis aórtica senil asintomática: ¿Existe algún tratamiento médico para enlentecer la progresión?
}

\author{
Asyntomatic senile degenerative aortic stenosis: Does any treatment show progression?
}

\begin{abstract}
Resumen
La estenosis aórtica (EAo) degenerativa senil es la causa más frecuente de EAo, y suele estar precedida por una esclerosis. Ambas presentan una clara relación con la edad. Presenta un largo periodo asintomático, y un mal pronóstico al hacerse sintomática, por lo que se indica la cirugía. El único tratamiento recomendado en asintomáticos es la profilaxis antibiótica para endocarditis. En el presente EOP se describe un escenario clínico del cual surge la siguiente pregunta: En pacientes con estenosis aórtica (EAo) degenerativa senil asintomática, ¿existe algún tratamiento médico que pueda detener ó enlentecer la progresión? Se realiza una búsqueda de la literatura para responderla, y de describe los estudios más relevantes detectados. Diversos hallazgos fisiopatológicos sugieren que las estatinas o los inhibidores de la enzima convertidora de angiotensina (IECA) podrían tener un papel A pesar de existir algunos estudios observacionales y aleatorizados pequeños, la evidencia no es concluyente acerca de la efectividad de un tratamiento médico para reducir la progresión de esta entidad.
\end{abstract}

\section{Abstract}

Degenerative senile aortic stenosis $(\mathrm{AoE})$ is the most frequent cause of $\mathrm{AoE}$, and it's usually preceded by sclerosis. Both present a clear relationship with age. Patients have a long asymptomatic period, and a bad prognosis when becoming symptomatic, for what surgery is indicated. The only treatment recommended in the asymptomatic patients is antibiotic prevention for endocarditis. This "patient oriented evidence" article (POE) describes a clinical scenario in which the following question arise: In patients with senile asymptomatic aortic (AoE) degenerative stenosis, can some medical treatment stop or slow the progression? The author searched the literature to respond his question and describes the more outstanding detected studies.

Diverse fisiopathologic discoverys suggest that statins and angiotensin converter enzyme inhibitors (ACEI) may have a role. In spite of some observational and randomized small studies, the evidence is not conclusive about the effectiveness of a medical treatment to reduce the progression of this entity.

Palabras clave: estenosis aórtica, pronóstico, estatinas, inhibidores de la enzima convertidora de angiotensina, ancianos. Key words: aortic stenosis, statins, angiotensin converter enzyme inhibitors, old adult.

Falconi M. Estenosis aórtica severa asintomática. ¿Existe algún tratamiento médico para enlentecer la progresión? Evid. actual. práct. ambul. 10(1):26-28. Ene-feb.2007.

\section{Escenario clínico}

Paciente de sexo femenino de 71 años, dislipémica, que presenta un soplo sistólico de larga data. Asintomática al interrogatorio. Un ecocardiograma doppler realizado recientemente muestra ventrículo izquierdo hipertrófico con función sistólica conservada, válvula aórtica calcificada con apertura reducida, un gradiente pico de 49 $\mathrm{mmHg}$ y un área de $1,1 \mathrm{~cm}^{2}$ (estenosis severa).

Un ecocardiograma realizado en 1999 mostró un ventrículo izquierdo normal con esclerosis valvular aórtica sin estenosis, y uno de 2002 ventrículo izquierdo levemente hipertrófico y válvula aórtica calcificada con estenosis leve (gradiente pico de $24 \mathrm{mmHg}$ y área de $\left.1,57 \mathrm{~cm}^{2}\right)$.

Frente a la clara evidencia de progresión de la enfermedad, la paciente pregunta sobre algún tratamiento que pueda detenerla ó retrasarla. Esta pregunta es habitual en estos casos, y no tenemos aún una respuesta a la misma.

\section{Pregunta que generó el caso}

En pacientes adultos con estenosis aórtica (EAo) degenerativa senil asintomática, ¿existe algún tratamiento médico que pueda detener ó enlentecer la progresión?

\section{Estrategia de búsqueda}

Se realizó una búsqueda en MEDLINE de publicaciones que hayan evaluado algún tipo de tratamiento médico destinado a modificar la progresión de la EAo degenerativa senil en estadio asintomático. Se utilizaron como palabras clave "Aortic stenosis and Treatment" y en base a los resultados se amplió la búsqueda a "Aortic stenosis and statins" y "Aortic stenosis and angiotensin converting enzyme inhibitors". Se evaluaron publicaciones en diversos idiomas de trabajos, observacionales ó ensayos clínicos controlados.

\section{Introducción}

La causa más común de EAo es la degenerativa senil, le siguen la bicúspide (congénita) y en mucha menor medida la reumática y otras causas.

La EAo degenerativa senil suele estar precedida por una esclerosis (si bien no todas las esclerosis progresan a estenosis), y ambas presentan una clara asociación con la edad: aproximadamente un
$50 \%$ de los pacientes mayores de 85 años presentan algún grado de alteración valvular aórtica (esclerosis en la mayoría, estenosis en aproximadamente el $3 \%){ }^{2}$

La EAo presenta un largo período asintomático, de buen pronóstico pero en el cual progresa en severidad, y un breve período sintomático en el que está indicado el reemplazo valvular porque el pronóstico es malo a mediano plazo (sobrevida promedio de 5 años en presencia de angor, tres años con síncope y dos años si el paciente presenta disnea ó insuficiencia cardíaca) ${ }^{3}$.

El único tratamiento recomendado en asintomáticos es la profilaxis antibiótica para endocarditis. No se ha demostrado aún otra medida terapéutica que modifique el pronóstico.

Recientes investigaciones revelan que el deterioro valvular no es consecuencia del mero desgaste mecánico: existe un proceso inflamatorio activo similar en algunos aspectos a la aterosclerosis, con infiltración de macrófagos, linfocitos T activados, moléculas de adhesión celular (E-selectina, ICAM-1, VCAM-1), metaloproteinasas, lipoproteínas, depósitos lipídicos, calcificación y áreas de osificación (ésta última en los estadios más avanzados). Los niveles de proteína $C$ reactiva se encuentran aumentados. También la presencia de enzima convertidora de angiotensina (ECA), angiontensina II y del receptor de angiotensina II locales están incrementados. $4,5,6,7$

La histología de la valvulopatía aórtica se diferencia de la aterosclerosis vascular en que no suele observarse proliferación de músculo liso y hay escasa presencia de células espumosas, mientras que el grado de calcificación es más extenso y precoz en la lesión valvular $^{5}$

Existe además una asociación de la esclerosis y estenosis con los factores de riesgo coronario (edad, sexo masculino, hipertensión arterial, tabaquismo, diabetes, niveles de colesterol, lipoproteína A) y alteraciones del metabolismo fosfocálcico (particularmente insuficiencia renal, dialítica ó no). ${ }^{8,9}$

En base a tales evidencias, surgen dos líneas de investigación: por un lado las estatinas y por otro lado los inhibidores de la enzima convertidora de angiotensina (IECA), con la hipótesis que podrían tener un efecto beneficioso retrasando la progresión de la EAo. 


\section{Resumen de la evidencia}

Se encontraron seis estudios observacionales y un ensayo clínico controlado aleatorizado con estatinas, un estudio observacional con estatinas e IECA y un estudio observacional con IECA. Se seleccionaron tres estudios con estatinas, el combinado (estatinas e IECA) y el estudio con IECA:

Novaro G, Tiong I, Pearce G y col. Effect of Hydroxymethylglutaryl Coenzyme A Reductase Inhibitors on the progression of calcific aortic stenosis. Circulation 2001;104:2205-2209

Objetivo: determinar si la terapia con estatinas enlentece la progresión de la EAo.

Diseño: estudio observacional de cohorte retrospectiva*

Ámbito: laboratorio de ecocardiografía, Cleveland Clinic Foundation (Ohio)

Población: 174 pacientes con EAo leve a moderada asintomática. Edad promedio $68 \pm 12$ años. Se incluyeron pacientes que tuvieran al menos dos ecocardiogramas separados por al menos 6 meses.

Medición de los resultados: cambios del gradiente pico, medio y área valvular.

Resultados: 57 pacientes recibían algún tipo de estatina. El grupo que recibía estatinas fue más añoso, con mayor porcentaje de diabéticos, hipertensos y enfermos coronarios. El grado de estenosis fue similar en ambos grupos. Durante $21 \pm 7$ meses de seguimiento la reducción del área valvular fue mayor en el grupo no tratado $\left(0,11 \pm 0,18 \mathrm{~cm}^{2}\right.$ vs. $0,06 \pm 0,16 \mathrm{~cm}^{2}$ al año; $\left.p=0,03\right)$, con una progresión $45 \%$ menor en los pacientes que recibieron estatinas. El tratamiento con estatinas fue un predictor independiente de menor progresión $(p=0,02)$. No se observó asociación de los niveles de colesterol sérico con el grado de progresión de la estenosis.

Conclusión de los autores: los pacientes tratados con estatinas tienen una menor progresión de la EAo que los no tratados. Se justifica la realización de un estudio prospectivo aleatorizado.

Shavelle D, Takasu J, Budoff M, y col. HMG CoA reductase inhibitor (statin) and aortic valve calcium. Lancet 2002;359:11251126

Objetivo: evaluar si existe asociación entre tratamiento con estatinas y el grado de calcificación valvular aórtica medido por Tomografía Computada con haz de electrones.

Diseño: estudio observacional de cohorte retrospectiva*

Ámbito: División Cardiología, Universidad de Washington.

Población: 65 pacientes con calcificación valvular aórtica, edad promedio $68 \pm 8$ años. Se incluyeron pacientes que tuvieran al menos dos tomografías separados por al menos 6 meses.

Medición de los resultados: medición del puntaje de calcio por los métodos de Agatston y volumétrico.

Resultados: 28 pacientes recibían algún tipo de estatina. El grupo que recibía estatinas tuvo mayor porcentaje de dislipémicos e hipertensos. A 2,3 $\pm 1,5$ años, el porcentaje de acumulación de calcio anualizado fue de $32 \%$ (Intervalo intercuartilo 1,2-74,4\%) en el grupo no tratado, comparado con $12,1 \%$ (Intervalo intercuartilo 0,1 $29 \%)(p=0,006)$, lo que representó una progresión $63 \%$ menor con estatinas. En total, $44 \%$ menos pacientes tuvieron progresión con estatinas ( $57 \%$ no tratados vs. $32 \%$ tratados, $p=0,043)$.

Conclusión de los autores: las estatinas pueden alterar favorablemente la progresión de la calcificación valvular aórtica. Se justifica la realización de un estudio prospectivo randomizado.
Cowell S, Newby D, Prescott $R$ y cols. A randomized trial of intensive lipid-lowering therapy in calcific aortic stenosis. Scotish Aortic Stenosis and Lipid Lowering Trial, Impact on Regression (SALTIRE). N Engl J Med 2005;352:2389-2397.

Objetivo: evaluar si el tratamiento con $80 \mathrm{mg}$ de atorvastatina puede retrasar la progresión de la EAo.

Diseño: estudio aleatorizado, doble ciego controlado con placebo.

Ámbito: estudio multicéntrico realizado en 10 hospitales de Reino Unido.

Población: 155 pacientes con EAo (119 grado leve a moderada, 36 de grado severo), 77 randomizados al grupo intervención y 78 a placebo.

Intervención: atorvastatina $80 \mathrm{mg}$ diarios (ó su equivalente en placebo) durante una media de 25 meses (7-36). La adherencia fue del $97 \%$.

Medición de los resultados: cambio en el jet de velocidad aórtico (medido con ecodoppler) y el puntaje de calcio (medido por tomografía)

Resultados: No hubo diferencias en las características basales. En el seguimiento el colesterol LDL fue significativamente menor en el grupo tratado $(63 \pm 23 \mathrm{mg} / \mathrm{dl}$ vs. $130 \pm 30 \mathrm{mg} / \mathrm{dl} ; \mathrm{p}<0,001)$. No hubo diferencias en el grado progresión de la velocidad del jet aór-

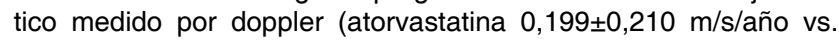

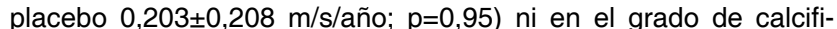
cación medido por tomografía (atorvastatina 1564ะ1956 U/año vs. $1648 \pm 1790$ U/año; $p=0,80)$. Se realizó un análisis de subgrupos, comparando la progresión en pacientes con estenosis leve a moderada (atorvastatina $0,17 \pm 0,21 \mathrm{~m} / \mathrm{s} /$ año vs. placebo $0,19 \pm 0,20$ $\mathrm{m} / \mathrm{s} / \mathrm{año} ; \mathrm{p}=0,57$ ) y en estenosis severa (atorvastatina $0,27 \pm 0,21$ $\mathrm{m} / \mathrm{s} / \mathrm{año} \mathrm{vs}$. placebo $0,27 \pm 0,23 ; \mathrm{p}=0,57)$, no encontrándose tampoco beneficios.

Conclusión de los autores: el tratamiento intensivo con estatinas no reduce la progresión de la EAo. Estudios aletaorizados controlados a mayor escala son necesarios para definir la utilidad de estas drogas.

Rosenhek R, Rader F, Loho N y cols. Statins but not angiotensinconverting enzyme inhibitors delay progression of aortic stenosis. Circulation 2004;110:1291-1295

Objetivo: evaluar el efecto de las estatinas e IECA en la progresión de la EAo.

Diseño: estudio observacional de cohorte retrospectiva*.

Ámbito: Departamento de Cardiología, Vienna General Hospital (Austria).

Población: 211 pacientes con EAo (edad media 70 \pm 10 años) Se incluyeron pacientes que tuvieran al menos dos ecocardiogramas separados por al menos 6 meses.

Medición de los resultados: cambios en el jet de velocidad aórtica, gradientes pico y medio y área valvular.

Resultados: de los 211 pacientes, 102 estaban tratados con estatinas, 50 con IECA y 32 con ambos. Los tratados con estatina fueron más añosos, con mayor prevalencia de mujeres, coronarios e hipercolesterolémicos; los tratados con IECA tuvieron mayor prevalencia de hipercolesterolemia, hipertensión, niveles de creatinina y superficie corporal. El seguimiento fue de $24 \pm 18$ meses.

La progresión de la velocidad del jet aórtico fue menor en el grupo que recibió estatinas $(0,10 \pm 0,41 \mathrm{~m} / \mathrm{s} / \mathrm{año})$ que en el que no recibió $(0,39 \pm 0,42 \mathrm{~m} / \mathrm{s} / \mathrm{año})(\mathrm{p}=0,0001)$, el análisis de subgrupos mostró que el beneficio fue similar en pacientes con estenosis leve a mo- 
derada y en pacientes con estenosis severa. No hubo asociación entre los niveles de colesterol y la tasa de progresión.

No hubo diferencias entre quienes recibieron IECA $(0,29 \pm 0,44$ $\mathrm{m} / \mathrm{s} / \mathrm{año})$ y quienes no $(0,35 \pm 0,44 \mathrm{~m} / \mathrm{s} / \mathrm{año})(p=0,29)$.

Conclusión de los autores: Los IECA no impresionan enlentecer la progresión de la EAo. Las estatinas tienen un efecto beneficioso tanto en estenosis leves a moderadas como en severas.

O'Brien K, Probstfield J, Caulfield M y cols. Angiotensin-converting enzyme inhibitors and change in aortic valve calcium. Arch Intern Med 2005;165:858-862.

Objetivo: evaluar si el tratamiento con IECA puede reducir la acumulación de calcio medida por tomografía (EBCT)

Diseño: estudio observacional de cohorte retrospectiva*

Ámbito: 4 hospitales de los Estados Unidos.

Población: 123 pacientes con EAo (edad media $68 \pm 9$ años) que tuvieran al menos dos tomografías separadas por al menos 6 meses

Medición de los resultados: puntaje de calcio medido por el método volumétrico.

Resultados: Los pacientes que recibieron IECA $(n=43)$ fueron significativamente más hipertensos, y con mayor puntaje de calcio basal (146,3 vs. 69,$5 ; p=0,001)$ El seguimiento fue de 2,6 $\pm 1,8$ años. Luego del ajuste por confundidores*, los pacientes que recibieron algún tipo de IECA tuvieron menor depósito de calcio que los que no lo recibieron: 11 (IC95\% 1,9-24,0) vs. 28,7 (IC95\% 18,9$38,9)(p=0,04)$ y menor porcentaje progresaron (42\% vs. $75 \%)$ $(\mathrm{p}<0,001)$, arrojando un Odds Ratio de progresión de 0,29 (IC95\% $0,11-0,75)(p=0,01)$

Conclusión de los autores: Existe una asociación significativa entre uso de IECA y menor depósito de calcio en EAo. Es necesario un estudio prospectivo randomizado.

\section{Comentarios y conclusiones:}

Las hipótesis del beneficio de estatinas e IECA en EAo son atractivas por las características fisiopatológicas de la lesión valvular. Sin embargo, muchas teorías apoyadas en mecanismos fisiopatológicos han sido descartadas con la evidencia de los estudios aleatorizados a gran escala. Todos los trabajos observacionales (incluso los cuatro no comentados aquí) orientan a un claro beneficio con estatinas. Sin embargo, el único ensayo aleatorizado controlado no muestra ninguna ventaja con su utilización.

Diversos argumentos pueden esgrimirse en ambos sentidos: si bien el seguimiento fue similar y ajustaron por confundidores ${ }^{*}$, persiste la presencia de sesgos* y confundidores no conocidos ó medidos en trabajos observacionales; por otro lado se requeriría mayor número de pacientes y seguimiento en el estudio controlado. Están en curso dos estudios clínicos controlados aleatorizados (ASTRONOMER y SEAS) que arrojarán mayor información en este tema. La evidencia con Ios IECA es menor y no hay estudios aleatorizados controlados con este tipo de drogas en EAo asintomática.

El reciente consenso de valvulopatías de la American Heart Association y American College of Cardiology, especifican claramente que fuera del cuidadoso control de la hipertensión arterial, profilaxis de fiebre reumática (en pacientes con valvulopatía aórtica reumática) y profilaxis antibiótica para endocarditis, no hay tratamiento médico recomendado para pacientes asintomáticos.

En conclusión, no hay evidencias que alguna medida terapéutica pueda retrasar la progresión de la EAo. La hipótesis más atractiva y estudiada es la utilización de estatinas, pero por el momento debieran ser empleadas, como es el caso de nuestra paciente, cuando hay otra indicación para las mismas (hipercolesterolemia, coronariopatía). No hay evidencias para recomendar IECA en esta población.

Ver glosario*

Mariano Falconi [ Médico de Planta, Servicio de Cardiología. Hospital Italiano de Buenos Aires ]

Recibido el el 12/11/2006. Aceptado para su publicación el el 10/02/2007

Referencias

1. Otto C, Burwash I, Legget M y col. Prospective Study of Asymptomatic Valvular Aortic Stenosis : Clinical, Echocardiographic, and Exercise Predictors of Outcome. Circulation 1997:95:2262-2270.

2. Stewart B, Siscovick D, Lind B y col. Clinical factors associated with calcific aortic valve disease. Cardiovascular Health Study. J Am Coll Cardiol 1997;29:630634.

3. Carabello B. Aortic Stenosis. N Engl J Med 2002;346:677-682.

4. Peltier M, Enriquez-Sarano M, Slama M, Tribouilloy Ch. New concepts on the physiopathology and therapy of aortic stenosis. Arch Mal Coeur Vaiss 2004:97:327-332

5. Cowell S, Newby D, Boon N, Elder A. Calcific aortic stenosis: same old story? Age and Ageing 2004;33:538-544

6. Galante A, Pietroiusti A, Vellini M y col. C-reactive protein is increased in patients with degenerative aortic valvular stenosis. J Am Coll Cardiol 2001;38:10781082

7. Helske S, Lindstedt K, Laine M y cols. Induction of local angiotensina II-producing systems in stenotic aortic valves. J Am Coll Cardiol 2004;44:1859-1866.

8. Cheitlin M. Pathophysiology of valvular aortic stenosis in the elderly. Am J Geriatr Cardiol 2003;12:173-177.

9. Scardi S, Cherubini A. E possibile prevenire la progressione della sclerosi e della stenosi valvolare aortica? Necessità di uno studio prospettico randomizzato. Ital Heart J Suppl 2005;6:403-412

10. Bonow RO, Carabello BA, Chatterjee K, de Leon AC Jr., Faxon DP, Freed MD, Gaasch WH, Lytle BW, Nishimura RA, O'Gara PT, O'Rourke RA, Otto CM, Shah PM, Shanewise JS. ACC/AHA 2006 guidelines for the management of patients with valvular heart disease: a report of the American College of Cardiology/American Heart Association Task Force on Practice Guidelines. J Am Coll Cardiol 48:e1-e148. 